\title{
Pteridófitas do Parque Estadual do Jacupiranga, SP, Brasil
}

\author{
Alexandre Salino ${ }^{1,3}$ e Thaís Elias Almeida ${ }^{1,2}$
}

Recebido em 13/06/2007. Aceito em 31/01/2008

\begin{abstract}
RESUMO - (Pteridófitas do Parque Estadual do Jacupiranga, SP, Brasil). Este trabalho refere-se ao levantamento da flora pteridofítica do Parque Estadual do Jacupiranga, localizado nos municípios de Barra do Turvo, Cajati, Cananéia, Eldorado, Iporanga e Jacupiranga, na região sul do Estado de São Paulo. As coletas foram realizadas em março e abril/2005 e os espécimes testemunho encontram-se depositados nos herbários BHCB, ESA, SPSF e UEC. Foram encontrados 212 táxons (207 espécies, uma subespécie e quatro variedades) distribuídas em 24 famílias e 65 gêneros. As famílias mais representativas foram Thelypteridaceae com 23 espécies e três variedades e Polypodiaceae com 23 espécies. Os gêneros mais ricos foram: Thelypteris com 22 espécies e três variedades e Asplenium com 18 espécies e uma variedade. Com relação à forma de vida, dos 212 táxons, 122 são terrestres, 65 epífitas, 17 rupícolas, três trepadeiras e cinco com mais de um hábitat. As espécies Diplazium rieddelianum Kunze, Stigmatopteris ulei (Christ) Sehnem e Thelypteris paranaensis Salino são aqui registradas pela primeira vez para o Estado de São Paulo.
\end{abstract}

Palavras-chave: Floresta Atlântica, florística, Jacupiranga, pteridófitas, vale do Ribeira

\begin{abstract}
Pteridophytes of Jacupiranga State Park, São Paulo State, Brazil). A floristic survey was carried out of the pteridophytes from Jacupiranga State Park, located in the municipal districts of Barra do Turvo, Cajati, Cananéia, Eldorado, Iporanga and Jacupiranga, in southern São Paulo state. Collecting was done in March and April/2005; voucher specimens are deposited in the BHCB, ESA, SPSF and UEC herbaria. Two hundred and twelve taxa (207 species, one subspecies and four varieties) distributed in 24 families and 65 genera were recorded. The most representative families were Thelypteridaceae with 23 species and three varieties and Polypodiaceae with 23 species. The richest genera were Thelypteris with 22 species and three varieties and Asplenium with 18 species and one variety. As regards life form, of the 212 taxa, 122 are terrestrial, 65 epiphytes, 17 lithophytes, three creepers and five with more than one habitat. The species Diplazium rieddelianum Kunze, Stigmatopteris ulei (Christ) Sehnem and Thelypteris paranaensis Salino are reported here for the first time in São Paulo state.
\end{abstract}

Key words: Atlantic Forest, floristic, Jacupiranga, pteridophytes, Ribeira Valley

\section{Introdução}

As pteridófitas são as plantas vasculares sem sementes, de ciclo de vida heteromórfico, com duas fases bem distintas: a fase gametofítica, inconspícua e efêmera, e a fase esporofítica, de maior porte e complexidade anatômica e período de vida mais longo. Ocorrem nos mais variados ambientes e hábitats, em condições bem distintas: dos ambientes árticos e alpinos das elevadas altitudes e latitudes até ambientes úmidos do interior das florestas tropicais; de situações sub-desérticas até as formações costeiras pantanosas. Entretanto, os ambientes de maior ocorrência das pteridófitas são aqueles tropicais bastante úmidos, que não apresentam períodos secos apreciáveis durante o ano (Page 1979a). A circunscrição de hábitat para determinada espécie de pteridófita normalmente é determinada pela associação de diversos fatores físicos, tais como profundidade do solo, $\mathrm{pH}$, drenagem, tipo de solo ou rocha, aeração do solo, grau de sombreamento e abrigo, regularidade do suprimento de água e da precipitação, umidade do ar, temperatura, tipo e duração da incidência luminosa (Page 1979b).

Essas plantas hoje estão incluídas em duas linhagens monofiléticas filogeneticamente distintas: as Licófitas e as Monilófitas (Pryer et al. 2004; Smith et al. 2006). A diversidade atualmente conhecida das pteridófitas é de 10.500 a 11.300 espécies, mas a diversidade estimada chega a 12.000-15.000 espécies (Roos 1996). Para o Brasil, Prado (1998) estima a ocorrência de 1.200-1.300 espécies, e cerca de 500-600 são conhecidas para o Estado de São Paulo. Além disso, Tryon \& Tryon (1982) consideram a região Sudeste e Sul do Brasil como um importante centro de endemismo do Neotrópico, com aproximadamente $40 \%$ das espécies da região endêmicas.

\footnotetext{
1 Universidade Federal de Minas Gerais, Departamento de Botânica, Instituto de Ciências Biológicas, C. Postal 486, $30120-970$ Belo Horizonte, MG, Brasil

2 Programa de Pós-Graduação em Biologia Vegetal, Universidade Federal de Minas Gerais

3 Autor para correspondência: salino@icb.ufmg.br
} 
A Mata Atlântica encontra-se em situação crítica de alteração dos ecossistemas naturais, estando entre os oito hotspots mais ricos e ameaçados do planeta (Myers et al. 2000). Hoje, o bioma está reduzido a menos de $8 \%$ de sua extensão original e seus domínios abrigam $70 \%$ da população e concentram as maiores cidades e os grandes pólos industriais do Brasil (MMA/SBF 2002).

O Estado de São Paulo apresentava-se coberto pela Mata Atlântica em 83,6\% de seu território. Restaram $12,16 \%$, concentrados, principalmente, no litoral e Vale do Ribeira (Fundação SOS Mata Atlântica/INPE 2005). Paradoxalmente, é nesse estado que se encontram os mais extensos remanescentes, bastante significativos para o bioma: representam 18\% da área de Mata Atlântica que restou no Brasil. Ainda assim, poucos levantamentos florísticos de pteridófitas já foram publicados para o Estado de São Paulo. Destacam-se os trabalhos mais recentes de Simabukuro (1994), Salino (1996), Salino \& Joly (2001), Athayde Filho et al. (2003), Prado (2004) e Boldrin \& Prado (2007).

Esse trabalho teve como objetivo realizar o levantamento da pteridoflora do Parque Estadual do Jacupiranga, contribuindo para o conhecimento da flora do Parque, do Vale do Ribeira e da Mata Attântica no Estado de São Paulo.

\section{Material e métodos}

Área de estudo - O Parque Estadual do Jacupiranga (PEJ) é um dos mais extensos remanescentes de Mata Atlântica, tendo aproximadamente 150.000 ha. Está localizado nos municípios de Barra do Turvo, Cajati, Cananéia, Eldorado, Iporanga e Jacupiranga, na região do Vale do Ribeira, sul do Estado de São Paulo. Apresenta uma grande amplitude altitudinal, de 10 a 1.310 metros. Entretanto, a maior parte da área do Parque está localizada na faixa altitudinal entre 700 e 900 metros, com poucas áreas abaixo dos 100 metros e apenas alguns picos isolados chegando a altitudes mais elevadas na Serra do Cadeado (Mário J. Nunes de Souza, comunicação pessoal). O PEJ foi criado em 1969 pelo decreto-lei Estadual no 145 de 8 de agosto de 1969. Localiza-se na Província Espeleológica do Vale do Ribeira, onde se encontra grande densidade de cavernas de médio porte. Há registro de seis grutas na área do Parque e possivelmente existe mais de uma dezena de outras pequenas cavidades com potencial turístico. A maior parte da superfície do Parque está localizada na Bacia do Rio Ribeira de Iguape. Pela importância na preservação do patrimônio natural, histórico e cultural, a região do Vale do Ribeira e a região litorânea sul do Estado de São Paulo foram reconhecidas como Reserva da Biosfera da Mata Atlântica e Sítio do Patrimônio Mundial Natural.
O parque apresenta as seguintes formações vegetacionais: Floresta Ombrófila Densa, Floresta Ombrófila Densa sobre afloramento calcáreo, Floresta sobre restinga, Restinga arenosa e Caxetal. Entretanto, a área do PEJ não é um contínuo florestal, visto que existem intensas pressões antrópicas das comunidades que vivem no seu interior e no entorno. Além disso, o $\mathrm{PEJ}$ é recortado por várias rodovias e estradas, sendo a Rodovia Regis Bittencourt (BR-116) a maior delas. A maioria das comunidades existentes dentro da área do parque só se estabeleceu após a abertura dessa rodovia. Outras são comunidades tradicionais, como as comunidades quilombolas e caiçaras. Além do patrimônio natural da Mata Atlântica, o PEJ abriga ainda sítios arqueológicos, como os sambaquis, e as cavernas, patrimônio geológico com bastante apelo turístico.

Amostragem e análise de dados - As coletas foram realizadas em março e abril/2005. Os exemplares foram coletados e preparados usando as técnicas usuais para pteridófitas. As coletas foram realizadas em todas as fitofisionomias presentes no parque, do nível do mar até aproximadamente $800 \mathrm{~m}$ de altitude. Foram feitas observações em campo e anotações sobre o hábito e a forma de vida de cada espécie. Além disso, em cada trilha percorrida dentro do PEJ, foi anotada a presença de todas as espécies observadas. As trilhas exploradas durante o trabalho de campo foram: Trilha do Araçá e Trilha do Rolado (consideradas aqui juntas devido à continuidade física), Trilha do Bugio, Trilha do Caxetal, Trilha do Evaristo, Trilha do Mirante do Angico, Trilha da Ressurgência ou Trilha da Água Grande, Trilha do Rio das Minas e Trilha do Rio das Ostras no Núcleo Caverna do Diabo; e Trilha do Aríete, Trilha da Cachoeira do Véu da Noiva, Trilha do Canal do Varadouro, Trilha do Cedro e Trilha próxima a Santa Maria, no Núcleo Cedro. As formações vegetacionais encontradas em cada trilha foram definidas em campo pela equipe de especialistas presentes: Dr. Ricardo Ribeiro Rodrigues (Universidade de São Paulo - ESALQ), Dr. Jorge Yoshio Tamashiro (UNICAMP) e Dr. Vinícius Castro Souza (Universidade de São Paulo - ESALQ).

Os espécimes testemunho encontram-se depositados nos herbários BHCB, ESA, SPSF e UEC - as siglas dos herbários estão de acordo com Holmgren et al. (1990). As espécies foram identificadas através de literatura específica e por comparação com material já determinado por especialistas existente no herbário do Departamento de Botânica - ICB - UFMG (BHCB). No caso de espécies de difícil determinação, duplicatas foram enviadas a especialistas para confirmação. Os táxons estão listados em ordem alfabética e o arranjo adotado para famílias e gêneros seguiu Moran \& Riba (1995), exceto para as famílias Gleicheniaceae e 
Vittariaceae, para as quais foram utilizadas as classificações genéricas de Ching (1940) e Crane (1997), respectivamente, e em Polypodiaceae adotou-se o gênero Serpocaulon, descrito recentemente por Smith et al. (2006). Para as abreviações dos nomes dos autores das espécies adotou-se o trabalho de Pichi-Sermolli (1996).

\section{Resultados e discussão}

No Parque Estadual do Jacupiranga foram encontrados 212 táxons infragenéricos, (207 espécies, uma subespécie e quatro variedades), distribuídos em 24 famílias e 65 gêneros (Tab. 1). As famílias mais ricas foram: Thelypteridaceae (23 espécies e três variedades), Polypodiaceae (23 espécies), Pteridaceae (20 spp.), Aspleniaceae (19 espécies e uma variedade), Hymenophyllaceae (16 spp.), Tectariaceae (14 spp.) e Lomariopsidaceae (13 spp.). Estas sete famílias abrangem aproximadamente $62 \%$ das espécies encontradas no parque. Os gêneros mais representativos foram: Thelypteris (22 espécies e três variedades), Asplenium (18 espécies e uma variedade), Elaphoglossum (11 spp.), Trichomanes (11 spp.), Blechnum (seis espécies e uma subespécie), Ctenitis (sete spp.), Diplazium (sete spp.), Pteris (sete spp.) e Selaginella (sete spp.). Dos 212 táxons encontrados, $122(57,5 \%)$ apresentam hábito terrestre, $65(30,6 \%)$ são epífitas, $17(8,1 \%)$ são rupícolas, três $(1,4 \%)$ são trepadeiras e cinco $(2,4 \%)$ apresentam hábito variado.

A flora de pteridófitas do Parque Estadual do Jacupiranga apresenta diversas peculiaridades, sendo que algumas das espécies encontradas são citadas aqui pela primeira vez para o Estado de São Paulo. São elas: Stigmatopteris ulei (Christ) Sehnem, conhecido antes apenas nos Estados de Santa Catarina e Paraná (Sehnem 1979; Moran 1991), Thelypteris paranaensis Salino, antes só citado para o Estado do Paraná (Salino 2002) e Diplazium rieddelianum (Bong. ex Kuhn) Kuhn ex C. Chr., citado anteriormente no Brasil apenas para Santa Catarina e Minas Gerais (Sehnem 1979). Além disso, o número de espécies encontrados no PEJ é bastante alto, e está relacionado à existência de enorme diversidade de condições físicas, climáticas e edáficas, às diferentes formações vegetacionais - desde formações de regiões montanas até as formações características da planície litorânea - e todos os diferentes microhábitats associados a essas condições.

Uma das espécies registradas no PEJ, Dicksonia sellowiana Hook., popularmente conhecida como xaxim, consta na Lista das Espécies da Flora Ameaçadas de Extinção no Estado de São Paulo na categoria "Vulnerável" (Estado de São Paulo 2004). Essa espécie apresenta amplo uso comercial e suas populações naturais encontram-se sob constante pressão de extrativismo. Outras sete espécies também encontradas na PEJ estão nessa lista, na categoria "Vulnerável". São elas: Elaphoglossum iguapense Brade, Elaphoglossum longifolium (Jacg.) J. Sm., Ctenitis anniesii (Rosenst.) Copel., Thelypteris concinna (Willd.) Ching, Thelypteris hatschbachii A.R. Sm., Thelypteris leprieurii (Hook.) R.M. Tryon e Anetium citrifolium (L.) Splitg.

O número de espécies encontrado no PEJ é um dos maiores já registrado em levantamentos da pteridoflora em áreas de conservação de Mata Atlântica. Comparando-se os dados aqui apresentados com outros levantamentos de pteridófitas observa-se a composição ímpar da pteridoflora do parque. Sylvestre (1997) encontrou na Reserva Ecológica de Macaé de Cima 80 espécies em uma área de 7.200 ha de Floresta Ombrófila Densa no Estado do Rio de Janeiro; Melo \& Salino (2007) encontraram 174 espécies em uma região de aproximadamente 3.600 ha de Floresta Ombrófila Densa e Floresta Ombrófila Mista na Serra da Mantiqueira, no Estado de Minas Gerais. Na Ilha do Mel (município de Paranaguá, Paraná), que apresenta uma diversidade de fitofisionomias similar ao Parque Estadual do Jacupiranga, Salino et al. (2005) encontraram, em 2.894 ha, 114 espécies.

A porcentagem de espécies epífitas encontradas no PEJ é menor do que o registrado em outros levantamentos realizados em áreas onde a Floresta Ombrófila Densa é a formação predominante; Dittrich et al. (2005) encontraram 62,9\% de epífitas nas espécies amostradas no Parque Estadual do Pico do Marumbi (PR); Sylvestre (1997) registrou 48,7\% de epífitas em seu levantamento da Reserva Ecológica de Macaé de Cima (RJ). Em outras áreas onde a Floresta Ombrófila Densa é apenas uma formação inserida dentro de um mosaico de formações vegetacionais o número de espécies epífitas diminui, como exemplificado pelo trabalho de Melo e Salino (2007) que encontraram 23,2\% de epífitas na APA Fernão Dias (Serra da Mantiqueira) e Salino et al. (2005), que encontraram apenas 19,29\% de epífitas dentre as 114 espécies registradas para a Ilha do Mel (PR).

A existência de ambientes antropizados na área do PEJ - como fazendas e áreas em estágio inicial de regeneração - e a presença de estradas e rodovias, facilitam a introdução de espécies exóticas. $\mathrm{Na}$ lista de espécies de pteridófitas do PEJ aparecem quatro espécies naturalizadas na região: Pteris vittata L., Macrothelypteris torresiana (Gaudich.) Ching, Thelypteris dentata (Forssk.) E.P. St. John e Diplazium petersenii (Kunze) Christ.

As formações vegetacionais encontradas em cada trilha estão listadas na Tab. 2. As trilhas onde foram observados os maiores números de espécies (Tab. 1) 
Tabela 1. Lista das espécies de pteridófitas ocorrentes no Parque Estadual do Jacupiranga, SP, Brasil, e lista de ocorrência das espécies por trilha. (Testem. $=$ Testemunho: $\mathrm{BHCB}=$ Herbário BHCB; $\mathrm{S}=$ Alexandre Salino). Hábito: $\mathrm{EP}=$ epífita; HE = hemiepífita; RU = rupícola; $\mathrm{TE}=$ terrestre; $\mathrm{TR}=$ trepadeira. Trilhas: $\mathrm{AN}=$ Trilha do Mirante do Angico; $\mathrm{AR}=$ Trilha do Araçá e Trilha do Rolado; $\mathrm{BU}=$ Trilha do Bugio; $\mathrm{CA}=$ Trilha do Caxetal; EV = Trilha do Evaristo; $\mathrm{MI}=$ Trilha do Rio das Minas; $\mathrm{OS}=$ Trilha do Rio das Ostras; RE = Trilha da Ressurgência ou Trilha da Água Grande (Núcleo Caverna do Diabo); AR = Trilha do Aríete; CE = Trilha do Cedro; SA = Trilha próxima a Santa Maria; VA = Trilha do Canal do Varadouro; VE = Trilha da Cachoeira do Véu da Noiva; (Núcleo Cedro).

\begin{tabular}{|c|c|c|c|c|c|c|c|c|c|c|c|c|c|c|c|}
\hline Família/Espécie & Testem. & Hábito & $\mathrm{AN}$ & $\mathrm{AR}$ & BU & $\mathrm{CA}$ & EV & MI & OS & $\mathrm{RE}$ & $\mathrm{AR}$ & $\mathrm{CE}$ & AS & VA & VE \\
\hline \multicolumn{16}{|l|}{ ASPLENIACEAE } \\
\hline Antigramma brasiliensis (Sw.) T. Moore & S 10211 & TE & & & & & & & & $\mathrm{X}$ & & & & & \\
\hline Asplenium abscissum Willd. & S 10226 & TE & & & $\mathrm{X}$ & & & & & $\mathrm{X}$ & & & & & \\
\hline A. alatum Willd. & S 10169 & RU & & & $\mathrm{X}$ & & & & & & & & & & \\
\hline A. auritum Sw. & S 10288 & EP & & $X$ & & $\mathrm{X}$ & & & & & & & & & \\
\hline A. cristatum Lam. & S 10096 & RU & & $\mathrm{X}$ & & & & & & & & & & & \\
\hline A. harpeodes Kunze & S 10203 & EP & & & & & & & & & $\mathrm{X}$ & & & & \\
\hline A. kunzeanum Klotzsch ex Rosenst. & S 10158 & TE & & $\mathrm{X}$ & $\mathrm{X}$ & & & & & & & & & & \\
\hline A. laetum Sw. & S 10261 & RU & & & & & & & $\mathrm{X}$ & & & & & & \\
\hline A. martianum C. Chr. & S 10321 & $\mathrm{EP}$ & & & & & & & & & & & $\mathrm{X}$ & & \\
\hline A. mourai Hieron. & S 10100 & RU & & $X$ & & & & & & & & & & & \\
\hline A. mucronatum C. Presl & S 10099 & EP & & $\mathrm{X}$ & & & & & & & & & & & \\
\hline A. pseudonitidum Raddi & S 10207 & TE & & & & & & & & & $X$ & & & & \\
\hline A. pteropus Kaulf. & S 10209 & EP & & $X$ & & & & & $X$ & & $\mathrm{X}$ & & & & \\
\hline A. pulchellum Raddi & S 10250 & RU & $X$ & & & & $\mathrm{X}$ & & & & & & & & \\
\hline A. raddianum Gaudich. & S 10204 & EP & & & & & & & & & $\mathrm{X}$ & & & & \\
\hline $\begin{array}{l}\text { A. radicans var. cirrhatum (Rich. ex } \\
\text { Willd.) Rosenst. }\end{array}$ & S 10206 & $\mathrm{TE}$ & & & $\mathrm{X}$ & & & & & & $\mathrm{X}$ & & & & \\
\hline A. scandicinum Kaulf. & S 10114 & $\mathrm{EP}$ & & $X$ & & & & & & & & & & & \\
\hline A. serra Langsd. \& Fisch. & S 10194 & EP & & & & & & & & & $\mathrm{X}$ & & & & \\
\hline A. serratum $\mathrm{L}$. & S 10324 & EP & & & & & & & & & & & & $\mathrm{X}$ & \\
\hline A. triquetrum N. Murak. \& R.C. Moran & S 10157 & RU & & & $\mathrm{X}$ & & & & & & & & & & \\
\hline \multicolumn{16}{|c|}{ BLECHNACEAE } \\
\hline $\begin{array}{l}\text { Blechnum binervatum subsp. acutum (Desv.) } \\
\text { R.M. Tryon \& Stolze }\end{array}$ & S 10201 & $\mathrm{EP}$ & & $\mathrm{X}$ & & & & & & & $\mathrm{X}$ & & & & \\
\hline B. brasiliense Desv. & S 10109 & TE & & $\mathrm{X}$ & & & & & & & & & & & \\
\hline B. cordatum (Desv.) Hieron. & S 10151 & TE & & & $\mathrm{X}$ & & & & & & & & & & \\
\hline B. occidentale $\mathrm{L}$. & S 10238 & TE & & & $\mathrm{X}$ & & & $\mathrm{X}$ & & $\mathrm{X}$ & & & & & \\
\hline B. polypodioides Raddi & S 10110 & TE & & $\mathrm{X}$ & & & & & & & & & & & \\
\hline B. sampaioanum Brade & S 10161 & TE & & & $\mathrm{X}$ & & & & & & & & & & \\
\hline B. serrulatum Rich. & S 10150 & TE & & & $\mathrm{X}$ & & & & & & & & & & \\
\hline Salpichlaena volubilis (Kaulf.) J. Sm. & S 10123 & TR & & $\mathrm{X}$ & & & & & & & & & & & \\
\hline \multicolumn{16}{|l|}{ CYATHEACEAE } \\
\hline Alsophila setosa Kaulf. & S 10198 & TE & & & & & & & & & $\mathrm{X}$ & & & & \\
\hline A. sternbergii (Sternb.) D.S. Conant & S 10233 & TE & & & & & & & & $\mathrm{X}$ & & & & & \\
\hline $\begin{array}{l}\text { Cyathea atrovirens (Langsd. \& Fisch.) } \\
\text { Domin }\end{array}$ & S 10323 & $\mathrm{TE}$ & & $\mathrm{X}$ & & & & & & & & & $\mathrm{X}$ & & \\
\hline C. corcovadensis (Raddi) Domin & S 10232 & TE & & & & & & & & $\mathrm{X}$ & & & & & \\
\hline C. delgadii Sternb. & S 10339 & TE & & & & & $\mathrm{X}$ & & & & & & & & \\
\hline C. hirsuta C. Presl & S 10152 & TE & & & $\mathrm{X}$ & & & & & & & & & & \\
\hline C. leucofolis Domin & S 10283 & TE & & & & & & $\mathrm{X}$ & $\mathrm{X}$ & & & & & & \\
\hline C. phalerata Mart. & S 10200 & TE & & & & & & & & & $\mathrm{X}$ & & & & \\
\hline \multicolumn{16}{|l|}{ DAVALLIACEAE } \\
\hline Nephrolepis biserrata (Sw.) Schott & S 10332 & TE & & & & & & & & & & & & $\mathrm{X}$ & \\
\hline N. pendula (Raddi) J. Sm. & S 10119 & $\mathrm{EP}$ & $\mathrm{X}$ & $\mathrm{X}$ & & & & & & & & & & & \\
\hline \multicolumn{16}{|l|}{ DENNSTAEDTIACEAE } \\
\hline Dennstaedtia cicutaria (Sw.) T. Moore & S 10306 & TE & & $\mathrm{X}$ & & & & & & & & & & & $\mathrm{X}$ \\
\hline D. $\operatorname{dissecta}(\mathrm{Sw}.) \mathrm{T}$. Moore & S 10340 & TE & & $\mathrm{X}$ & & & $X$ & & & & & & & & \\
\hline Lindsaea arcuata Kunze & S 10191 & TE & & & & & & & & & $\mathrm{X}$ & & & & \\
\hline L. lancea (L.) Bedd. & S 10290 & TE & & $\mathrm{X}$ & & $\mathrm{X}$ & & & & & & & & & \\
\hline L. quadrangularis Raddi & S 10322 & TE & & & & $\mathrm{X}$ & & & & & & & $\mathrm{X}$ & & \\
\hline L. virescens $\mathrm{Sw}$ & S 10213 & TE & & & & & & & & $\mathrm{X}$ & & & & & \\
\hline Pteridium arachnoideum (Kaulf.) Maxon & S 10346 & TE & & & & & & & & $\mathrm{X}$ & & & & & \\
\hline Saccoloma inaequale (Kunze) Mett. & S 10329 & TE & & & & & & & & & & & & $\mathrm{X}$ & \\
\hline \multicolumn{16}{|l|}{ DICKSONIACEAE } \\
\hline Dicksonia sellowiana Hook. & S 10188 & TE & & & & & & & & & $\mathrm{X}$ & & & & \\
\hline
\end{tabular}


Tabela 1 (continuação)

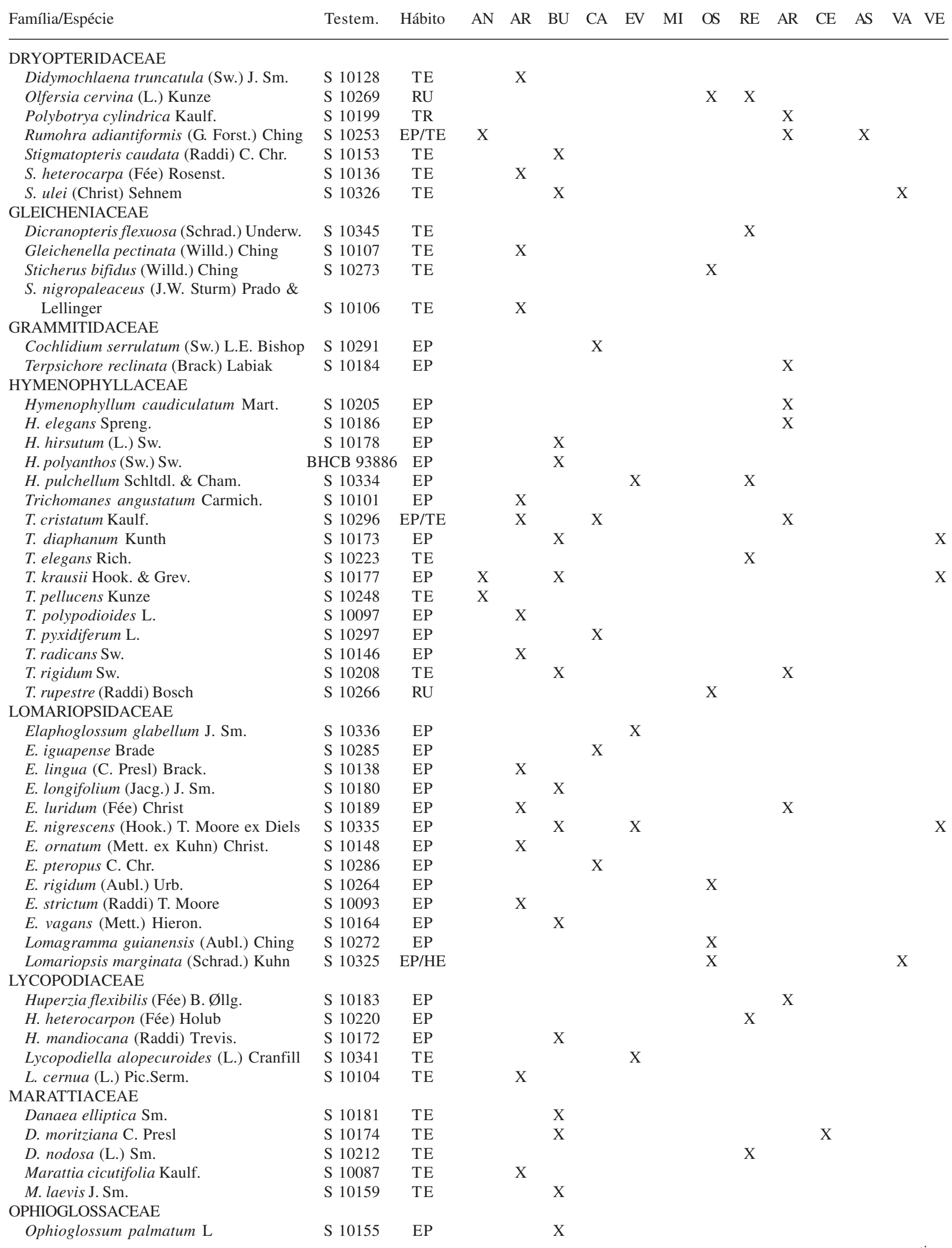


Tabela 1 (continuação)

\begin{tabular}{|c|c|c|c|c|c|c|c|c|c|c|c|c|c|c|c|}
\hline Família/Espécie & Testem. & Hábito & AN & $\mathrm{AR}$ & $\mathrm{BU}$ & $\mathrm{CA}$ & $\mathrm{EV}$ & MI & OS & $\mathrm{RE}$ & $\mathrm{AR}$ & $\mathrm{CE}$ & AS & VA & VE \\
\hline \multicolumn{16}{|l|}{ OSMUNDACEAE } \\
\hline Osmunda cinnamomea $\mathrm{L}$. & S 10344 & TE & & & $\mathrm{X}$ & & & & & & & & & & \\
\hline$O$. regalis $\mathrm{L}$. & S 10343 & TE & & & $\mathrm{X}$ & & & & & & & & & & \\
\hline \multicolumn{16}{|l|}{ POLYPODIACEAE } \\
\hline Campyloneurum acrocarpon Fée & S 10298 & EP & & & & $X$ & & & & & & & & & \\
\hline C. minus Fée & S 10134 & $\mathrm{EP} / \mathrm{RU}$ & & $\mathrm{X}$ & & & & & & & & & & $\mathrm{X}$ & \\
\hline C. nitidum (Kaulf.) C. Presl & S 10108 & EP & & $X$ & & & & & & & & & & & \\
\hline C. rigidum $\mathrm{J} . \mathrm{Sm}$. & S 10287 & $\mathrm{EP}$ & & $\mathrm{X}$ & & $\mathrm{X}$ & & & & & & & & & \\
\hline $\begin{array}{l}\text { Dicranoglossum furcatum (L.) J. Sm. } \\
\text { Tryon \& R.M. Tryon }\end{array}$ & S 10268 & $\mathrm{EP}$ & & & & & & & $\mathrm{X}$ & & & & & & \\
\hline Microgramma geminata (Schrad.) A.F. & S 10239 & $\mathrm{EP}$ & & & & & & $\mathrm{X}$ & & & & & & & \\
\hline M. percussa (Cav.) de la Sota & S 10084 & EP & & $X$ & & & & & & & & & & & \\
\hline M. squamulosa (Kaulf.) de la Sota & S 10103 & EP & & $\mathrm{X}$ & & & & & & & & & & & \\
\hline M. tecta (Kaulf.) Alston & S 10145 & EP & & $\mathrm{X}$ & & & & & & & & & & & \\
\hline M. vaccinniifolia (Langsd. \& Fisch.) Copel. & S 10124 & EP & & $\mathrm{X}$ & & & & & & & & & & & \\
\hline Niphidium crassifolium (L.) Lellinger & S 10131 & $\mathrm{EP}$ & & $\mathrm{X}$ & & & & & & & & & & & \\
\hline Pecluma recurvata (Kaulf.) M.G. Price & S 10083 & EP & & $\mathrm{X}$ & & & & & & & & & & & \\
\hline P. robusta (Fée) M. Kessler \& A.R. Sm. & S 10301 & TE & & & & & & & & & & & & & $\mathrm{X}$ \\
\hline P. truncorum (Lindm.) M.G. Price & S 10082 & EP & & $\mathrm{X}$ & & & & & & & & & & & \\
\hline Pleopeltis astrolepis (Liebm.) E. Fourn. & S 10125 & $\mathrm{EP}$ & & $X$ & & & & & & & & & & & \\
\hline P. pleopeltifolia (Raddi) Alston & S 10244 & $\mathrm{EP}$ & & & & & & $X$ & & & & & & & \\
\hline Polypodium chnoophorum Kunze & S 10196 & TE & & & $X$ & & & & & & $\mathrm{X}$ & & & & \\
\hline P. hirsutissimum Raddi & S 10102 & $\mathrm{EP}$ & & $\mathrm{X}$ & & & & & & & & & & & \\
\hline $\begin{array}{l}\text { Serpocaulon catharinae (Langsd. \& } \\
\text { Fisch.) A.R. Sm. }\end{array}$ & S 10129 & TE & & $\mathrm{X}$ & & & & & & & & & & & \\
\hline S. fraxinifolium (Jacq.) A.R. Sm. & S 10275 & EP & & $\mathrm{X}$ & & & & & $\mathrm{X}$ & & & & & & \\
\hline $\begin{array}{l}\text { S. meniscifolium (Langsd. \& Fisch.) } \\
\text { A.R.Sm. }\end{array}$ & S 10217 & EP & & & & & & & & $X$ & & & & & \\
\hline S. triseriale (Sw.) A.R. Sm. & S 10333 & EP & & & & & & $\mathrm{X}$ & & & & & & $\mathrm{X}$ & \\
\hline S. vacillans (Link) A.R. Sm. & S 10170 & EP & & & $\mathrm{X}$ & & & & & & & & & & \\
\hline \multicolumn{16}{|l|}{ PSILOTACEAE } \\
\hline Psilotum nudum (L.) P. Beauv. & S 10249 & TE & $X$ & & & & & & & & & & & & \\
\hline \multicolumn{16}{|c|}{ PTERIDACEAE } \\
\hline Acrostichum danaeifolium Langsd. \& Fisch. & S 10331 & TE & & & & & & & & & & & & $\mathrm{X}$ & \\
\hline Adiantopsis radiata $(\mathrm{L}$.$) Fée$ & S 10117 & TE & & $\mathrm{X}$ & & & & & & & & & & & \\
\hline Adiantum abscissum Schrad. & S 10317 & TE & & & & & & & & $\mathrm{X}$ & & & & & $\mathrm{X}$ \\
\hline A. curvatum Kaulf. & S 10318 & $\mathrm{TE}$ & & & & & & & $\mathrm{X}$ & $X$ & & & & & $\mathrm{X}$ \\
\hline A. lorentzii Hieron. & S 10319 & RU & & & & & & & & & & & & & $\mathrm{X}$ \\
\hline A. pentadactylon Langsd. \& Fisch. & S 10116 & TE & & $\mathrm{X}$ & & & & & & & & & & & \\
\hline A. raddianum C. Presl & S 10315 & TE & & & & & & & $\mathrm{X}$ & & & & & & $\mathrm{X}$ \\
\hline A. terminatum Kunze ex Miq. & S 10270 & TE & & & & & & & $\mathrm{X}$ & & & & & & \\
\hline $\begin{array}{l}\text { Doryopteris concolor (Langsd. \& Fisch.) } \\
\text { Kuhn }\end{array}$ & S 10303 & RU & & & & & & & & & & & & & $\mathrm{X}$ \\
\hline D. nobilis (T. Moore) C. Chr. & S 10316 & TE & & & & & & & & & & & & & $\mathrm{X}$ \\
\hline D. pentagona Pic.Serm. & S 10216 & TE/RU & & $\mathrm{X}$ & & & & & & $\mathrm{X}$ & & & & & \\
\hline D. sagittifolia (Raddi) J. Sm. & S 10327 & RU & & & & & & & & & & & & $\mathrm{X}$ & \\
\hline Pityrogramma calomelanos (L.) Link & S 10241 & TE & & & & & & $\mathrm{X}$ & & & & & & & \\
\hline Pteris altissima Poir. & S 10091 & TE & & $\mathrm{X}$ & & & & & & & & & & & \\
\hline P. decurrens C. Presl & S 10313 & TE & & & $X$ & & & & & $\mathrm{X}$ & & & & & $\mathrm{X}$ \\
\hline P. deflexa Link & S 10252 & TE & $\mathrm{X}$ & & & & & & & $\mathrm{X}$ & $\mathrm{X}$ & & & & \\
\hline P. plumula Desv. & S 10276 & TE & & & & & & & $\mathrm{X}$ & & & & & & \\
\hline P. schwackeana Christ. & S 10307 & TE & & & & & & & & & & & & & $\mathrm{X}$ \\
\hline P. splendens Kaulf. & S 10197 & $\mathrm{TE}$ & & & & & & & & & $\mathrm{X}$ & & & & \\
\hline P. vittata $\mathrm{L}$. & S 10247 & TE & $\mathrm{X}$ & & & & & & & & & & & & \\
\hline \multicolumn{16}{|l|}{ SCHIZAEACEAE } \\
\hline Actinostachys pennula (Sw.) Hook. & ЗНCB 93085 & TE & & & & & & & & & & & & $X$ & \\
\hline Anemia phyllitidis (L.) Sw. & S 10118 & TE & & $\mathrm{X}$ & & & & & & & & & & & \\
\hline A. raddiana Link & S 10210 & RU & & & & & & & & $\mathrm{X}$ & & & & & \\
\hline Lygodium volubile $\mathrm{Sw}$. & S 10242 & TR & & & & & & $\mathrm{X}$ & $\mathrm{X}$ & & & & & & \\
\hline Schizaea fluminensis Miers ex J.W. Sturm & S 10292 & TE & & & & $\mathrm{X}$ & & & & & & & & & \\
\hline
\end{tabular}


Tabela 1 (continuação)

\begin{tabular}{|c|c|c|c|c|c|c|c|c|c|c|c|c|c|c|c|}
\hline Família/Espécie & Testem. & Hábito & AN & $\mathrm{AR}$ & $\mathrm{BU}$ & $\mathrm{CA}$ & $\mathrm{EV}$ & MI & OS & $\mathrm{RE}$ & $\mathrm{AR}$ & $\mathrm{CE}$ & AS & VA & VE \\
\hline \multicolumn{16}{|l|}{ SELAGINELLACEAE } \\
\hline Selaginella contigua Baker & S 10193 & TE & & & & & & & & & $\mathrm{X}$ & & & & \\
\hline S. decomposita Spring & S 10337 & TE & & & & & $\mathrm{X}$ & & & & $\mathrm{X}$ & & & & \\
\hline S. flexuosa Spring & S 10305 & RU & & & & & & & & & & & & & $\mathrm{X}$ \\
\hline S. macrostachya (Spring) Spring & S 10246 & TE & $\mathrm{X}$ & & & & & & & & & & & & \\
\hline S. microphylla (Kunth) Spring & S 10260 & RU & & & & & & & $X$ & & & & & & \\
\hline S. muscosa Spring & S 10147 & TE & & $\mathrm{X}$ & $\mathrm{X}$ & & & & & & & & & & \\
\hline S. sulcata (Desv. ex Poir) Spring ex Mart. & S 10304 & TE & & $\mathrm{X}$ & & & & & & & & & & & $\mathrm{X}$ \\
\hline \multicolumn{16}{|l|}{ TECTARIACEAE } \\
\hline Ctenitis anniesii (Rosenst.) Copel. & S 10219 & TE & & & & & & & & $\mathrm{X}$ & & & & & \\
\hline C. aspidioides (C. Presl) Copel. & S 10228 & TE & & & & & & & & $\mathrm{X}$ & & & & & \\
\hline C. deflexa (Kaulf.) Copel. & S 10120 & TE & & $X$ & & & & & & & & & & & \\
\hline C. distans (Brack.) Ching & S 10135 & TE & & $\mathrm{X}$ & & & & & & & & & & & \\
\hline C. falciculata (Raddi) Ching & S 10215 & TE & & $\mathrm{X}$ & $\mathrm{X}$ & & & & & $\mathrm{X}$ & & & & & \\
\hline C. pedicellata (Christ) Copel. & S 10142 & TE & & $\mathrm{X}$ & & & & & & & & & & & \\
\hline C. submarginalis (Langsd. \& Fisch.) Ching & S 10312 & TE & & & & & & & $\mathrm{X}$ & $\mathrm{X}$ & & & & & \\
\hline Lastreopsis amplissima (C. Presl) Tindale & S 10090 & TE & & $\mathrm{X}$ & & & & & & & & & & & \\
\hline L. effusa (Sw.) Tindale & S 10237 & TE & & & & & & $\mathrm{X}$ & & $\mathrm{X}$ & & & & & \\
\hline $\begin{array}{l}\text { Megalastrum abundans (Rosenst.) A.R. } \\
\text { Sm. \& R.C. Moran }\end{array}$ & S 10160 & $\mathrm{TE}$ & & & $\mathrm{X}$ & & & & & & & & & & \\
\hline $\begin{array}{l}\text { M. canescens (Kunze ex Mett.) A.R. Sm. } \\
\text { \& R.C. Moran }\end{array}$ & S 10330 & TE & & & & & & & & & $\mathrm{X}$ & & & $X$ & \\
\hline $\begin{array}{l}\text { M. connexum (Kaulf.) A.R. Sm. \& } \\
\text { R.C. Moran }\end{array}$ & S 10267 & TE & & & $\mathrm{X}$ & & & & $\mathrm{X}$ & $\mathrm{X}$ & & & & & \\
\hline Tectaria incisa Cav. & S 10141 & TE & & $\mathrm{X}$ & & & & & & & & & & & \\
\hline T. pilosa (Fée) R.C. Moran & S 10132 & TE & & & & & & & & & & & & & \\
\hline \multicolumn{16}{|l|}{ THELYPTERIDACEAE } \\
\hline $\begin{array}{l}\text { Macrothelypteris torresiana (Gaudich.) } \\
\text { Ching }\end{array}$ & S 10243 & $\mathrm{TE}$ & & & & & & $\mathrm{X}$ & & & & & & & \\
\hline Thelypteris amambayensis (Christ) Ponce & S 10168 & TE & & & $\mathrm{X}$ & & & & & & & & & & \\
\hline T. concinna (Willd.) Ching & S 10309 & TE & & & & & & & & & & & & & $\mathrm{X}$ \\
\hline $\begin{array}{l}\text { T. decussata var. brasiliensis (C. Chr.) } \\
\text { A.R. Sm. }\end{array}$ & S 10154 & $\mathrm{TE}$ & & & $\mathrm{X}$ & & & & & & & & & & \\
\hline T. dentata (Forssk.) E.P. St. John & S 10314 & TE & & & & & & & & & & & & & $\mathrm{X}$ \\
\hline T. devolvens (Baker) C.F. Reed & S 10278 & $\mathrm{TE}$ & & & & & & & $\mathrm{X}$ & & & & & & \\
\hline T. hatschbachii A.R. Sm. & S 10192 & TE & & & & & & & & & $\mathrm{X}$ & & & & \\
\hline T. interrupta (Willd.) K. Iwats & S 10299 & TE & & & & $X$ & & & & & & & & & \\
\hline T. leprieurii (Hook.) R.M. Tryon & S 10225 & TE & & & & & & & & $\mathrm{X}$ & & & & & \\
\hline T. longifolia (Desv.) R.M. Tryon & S 10300 & TE & & & & $\mathrm{X}$ & & & & & & & & & \\
\hline $\begin{array}{l}\text { T. lugubris (Mett.) R.M. Tryon \& } \\
\text { A.F. Tryon }\end{array}$ & S 10320 & $\mathrm{TE}$ & & & & & & & $\mathrm{X}$ & & & & $\mathrm{X}$ & & \\
\hline T. maxoniana A.R. Sm. & S 10166 & TE & & & $\mathrm{X}$ & & & & $\mathrm{X}$ & & & & & & \\
\hline T. metteniana Ching & S 10262 & RU & & & & & & & $\mathrm{X}$ & & & & & & \\
\hline T. opposita (Vahl) Ching & S 10105 & TE & & $\mathrm{X}$ & & & & & $\mathrm{X}$ & & & & & & \\
\hline T. pachyrhachis (Kunze ex Mett.) Ching & S 10308 & TE & & & & & & & & & & & & & $\mathrm{X}$ \\
\hline T. paranaensis Salino & S 10140 & TE & & $\mathrm{X}$ & & & & & & & & & & & \\
\hline T. patens (Sw.) Small & S 10277 & TE & & & & & & & $\mathrm{X}$ & & & & & & \\
\hline T. polypodioides (Raddi) C.F. Reed & S 10229 & TE & & & & & & & $\mathrm{X}$ & $\mathrm{X}$ & & & & & \\
\hline T. ptarmica var. asplenioides (Sw.) Ponce & S 10279 & RU & & & & & & & $\mathrm{X}$ & & & & & & \\
\hline T. ptarmica (Mett.) C.F. Reed var. ptarmica & S 10311 & RU & & & & & & & & & & & & & $\mathrm{X}$ \\
\hline T. raddii (Rosenst.) Ponce & S 10133 & TE & & $\mathrm{X}$ & & & & & & & & & & & \\
\hline T. rivularioides (Fée) Abbiatti & S 10182 & TE & & & $\mathrm{X}$ & & & & & & & & & & \\
\hline T. serrata (Cav.) Alston & S 10149 & TE & & & $\mathrm{X}$ & & & & & & & & & & \\
\hline T. scabra (C. Presl) Lellinger & Observado & TE & $\mathrm{X}$ & & & & & & & & & & & & \\
\hline T. tenerrima (Fée) C.F. Reed & S 10310 & TE & & & & & & & & & & & & & $\mathrm{X}$ \\
\hline T. vivipara (Raddi) C.F. Reed & S 10265 & TE & & $\mathrm{X}$ & & & $\mathrm{X}$ & & $\mathrm{X}$ & & & & & & \\
\hline \multicolumn{16}{|l|}{ VITTARIACEAE } \\
\hline Anetium citrifolium (L.) Splitg. & S 10293 & EP & & & & $X$ & & & & & & & & & \\
\hline Polytaenium cajenense (Desv.) Benedict & S 10259 & $\mathrm{EP}$ & & & & & & & $\mathrm{X}$ & & & & & & \\
\hline P. lineatum (Sw.) J. Sm. & S 10095 & $\mathrm{EP}$ & & $X$ & & & & & & & & & & & \\
\hline Radiovittaria stipitata (Kunze) E.H. Crane & S 10295 & EP & & & & $\mathrm{X}$ & & & $\mathrm{X}$ & $\mathrm{X}$ & & & & & \\
\hline
\end{tabular}




\begin{tabular}{|c|c|c|c|c|c|c|c|c|c|c|c|c|c|c|c|}
\hline Família/Espécie & Testem. & Hábito & AN & $\mathrm{AR}$ & $\mathrm{BU}$ & $\mathrm{CA}$ & $\mathrm{EV}$ & MI & OS & $\mathrm{RE}$ & $\mathrm{AR}$ & $\mathrm{CE}$ & AS & VA & VE \\
\hline Vittaria graminifolia Kaulf. & BHCB 93885 & $5 \mathrm{EP}$ & & & & & & & & & & $\mathrm{X}$ & & & \\
\hline V. lineata $(\mathrm{L}.) \mathrm{Sm}$. & S 10081 & EP & & $\mathrm{X}$ & & $\mathrm{X}$ & & & & & & & & & \\
\hline \multicolumn{16}{|l|}{ WOODSIACEAE } \\
\hline Diplazium ambiguum Raddi & S 10230 & TE & & & & & $\mathrm{X}$ & & & $X$ & & & & & \\
\hline D. cristatum (Desr.) Alston & S 10143 & TE & & $\mathrm{X}$ & & & & & & & & & & & \\
\hline D. expansum Willd. & S 10224 & TE & & $\mathrm{X}$ & $\mathrm{X}$ & & & & & $X$ & & & & & \\
\hline D. lindbergii (Mett.) Christ & S 10162 & TE & & & $X$ & & & & & & & & & & $\mathrm{X}$ \\
\hline D. petersenii (Kunze) Christ & S 10111 & TE & & $\mathrm{X}$ & & & & & & & & & & & \\
\hline D. plantaginifolium (L.) Urb. & S 10126 & TE & & $X$ & & & & & & & & & & & \\
\hline $\begin{array}{l}\text { D. riedelianum (Bong. ex Kuhn) Kuhn } \\
\text { ex C. Chr. }\end{array}$ & S 10342 & $\mathrm{TE}$ & & & $\mathrm{X}$ & & & & & & & & & & \\
\hline Hemidictyum marginatum (L.) C. Presl & S 10280 & TE & & & & & & & $\mathrm{X}$ & & & & & & \\
\hline
\end{tabular}

foram as Trilhas do Araçá e do Rolado (ARA) com 69 espécies, Trilha do Bugio (BUG) com 42 spp., Trilha do Rio das Ostras (OST) com 31 spp., Trilha da Ressurgência (RES) com 29 spp. e Trilha do Aríete (ARI) com 27 espécies. Essas trilhas são as que se localizam em áreas de Floresta Ombrófila Densa, sendo que a vegetação das trilhas ARA, BUG e RES está associada a regiões de afloramento calcáreo. Nas trilhas que apresentam formações mais litorâneas foi registrada uma menor diversidade, porém nessas foram encontradas espécies que não ocorreram em nenhum outro ambiente, como Asplenium serratum L., Actinostachys pennula (Sw.) Hook., Schizaea fluminensis Miers ex J.W. Sturm, Anetium citrifolium (L.) Splitg. e Acrostichum danaeifolium Langsd. \& Fisch. O menor número de espécies pode estar associado estado de conservação diferente, ao tipo de substrato existente ou a uma diferente condição de umidade, influenciada pelo aumento dos ventos e/ou a salinidade.

Tabela 2. Trilhas percorridas e as respectivas formações vegetacionais encontradas. CAX = Caxetal; FOD = Floresta Ombrófila Densa; FODC = Floresta Ombrófila Densa sobre afloramento calcáreo; FSR $=$ Floresta Sobre Restinga RA $=$ Restinga Arenosa.

Trilha

Trilha do Araçá e do Rolado

Trilha do Bugio

Trilha do Caxetal

Trilha do Evaristo

Trilha do Mirante do Angico

Trilha da Ressurgência ou da Água Grande

Trilha do Rio das Minas

Trilha do Rio das Ostras

Trilha do Aríete

Trilha do Canal do Varadouro

Trilha da Cachoeira do Véu da Noiva

Trilha do Cedro

Trilha próxima a Santa Maria
Formação vegetacional
FODC

FODC

CAX/FSR

FOD

FODC

FODC

FOD

FOD

FOD

FOD / RA

FOD

FOD

FOD / FSR
Quanto à distribuição geográfica das espécies encontradas, aproximadamente a metade delas (101 espécies - 47,6\%) apresenta ampla distribuição na região Neotropical; 60 (28,3\%) estão restritas ao Brasil, e dessas, 40 (18,9\%) são endêmicas da Região Sudeste e Sul do Brasil (incluindo a região sul do Estado da Bahia).

Os resultados apresentados nesse estudo mostram a necessidade urgente de realizar inventários florísticos nas regiões remanescentes de Mata Atlântica, visto que as informações disponíveis sobre ocorrência e distribuição geográfica de espécies de pteridófitas são escassas na literatura e nas coleções. Além disso, essas informações possibilitam o manejo sustentável das Unidades de Conservação, contribuindo para a preservação da riqueza de um dos biomas mais ameaçados do planeta.

\section{Agradecimentos}

Aos professores Dr. Ricardo Ribeiro Rodrigues, Dr. Vinícius Castro Souza, Dr. Jorge Tamashiro, e à Natalia Ivanauskas, pela oportunidade de participar do curso "Taxonomia de campo de espécies vegetais", oferecido pela Escola Superior de Agricultura Luiz de Queiroz da Universidade de São Paulo e pela Universidade Estadual de Campinas; aos alunos que participaram do curso pela ajuda nas coletas e no trabalho de campo; ao Dr. Jefferson Prado, pela identificação de espécies do gênero Adiantum; ao Instituto Florestal de São Paulo, pelo apoio; ao CNPq, pela concessão da Bolsa Produtividade em Pesquisa ao primeiro autor.

\section{Referências bibliográficas}

Athayde Filho, F.P.; Pereira, V.S.; Smidt, E.C. \& Nonato, F.R. 2003. Pteridófitas do Parque Estadual da Ilha Anchieta (PEIA), Ubatuba, São Paulo, Brasil. Bradea 9: 55-66. 
Boldrin, A.H.L. \& Prado, J. 2007. Pteridófitas terrestres e rupícolas do Forte dos Andradas, Guarujá, São Paulo, Brasil. Boletim de Botânica da Universidade de São Paulo 25: 1-69.

Ching, R.C. 1940. On the genus Gleichenia Smith. Sunyatsenia 5 269-289.

Crane, E.H. 1997. A revised circunscription of the genera of the fern family Vittariaceae. Systematic Botany 22: 509-517.

Dittrich, V.A.O.; Waechter, J.L. \& Salino, A. 2005. Species richness of pteridophytes in a montane Atlantic rain Forest plot of Southern Brazil. Acta Botanica Brasilica 19: 519-525.

Estado de São Paulo. 2004. Resolução SMA - 48, de 22 de setembro de 2004. Dispõe sobre as espécies da flora ameaçadas de extinção no Estado de São Paulo. Diário Oficial do Estado de São Paulo, São Paulo, Imprensa Oficial.

Fundação SOS Mata Atlântica/INPE. 2005. Atlas dos remanescentes florestais da Mata Atlântica no período de 2000-2005. São Paulo, Fundação SOS Mata Atlântica/INPE.

Holmgren, P.K.; Holmgren, N.H. \& Barnett, L.C. 1990. Index Herbariorum. Part I. The herbaria of the world. $8^{\text {th }}$ ed New York, The New York Botanical Garden.

Labiak, P.H. \& Prado, J. 2003. Os gêneros Ceradenia, Cochlidium e Grammitis no Brasil. Hoehnea 30: 243-283.

Melo, L.C.N. \& Salino, A. 2007. Pteridófitas em fragmentos florestais da APA Fernão Dias, Minas Gerais, Brasil. Rodriguésia 59: 207-220.

MMA/SBF. 2002. Biodiversidade Brasileira: Avaliação e identificação de áreas e ações prioritárias para a conservação, utilização sustentável e repartição de benefícios da biodiversidade brasileira. Brasília, Ministério do Meio Ambiente/SBF.

Moran, R.C. 1991. Monograph of the neotropical fern genus Stigmatopteris (Dryopteridaceae). Annals of the Missouri Botanical Garden 78: 857-914.

Moran, R.C. \& Riba, R. (eds.). 1995. Psilotaceae a Salviniaceae. In: G. Davidse; M. Souza \& S. Knapp (eds.). Flora Mesoamericana. v.1. México, Universidad Nacional Autónoma de México.

Myers, N.; Mittermeier, R.A.; Mittermeier, C.G.; Fonseca, G.A.B. \& Kent, J. 2000. Biodiversity hotspots for conservation priorities. Nature 403: 853 - 858 .

Page, C.N. 1979a. The diversity of ferns: an ecological perspective. Pp. 10-56. In: A.F. Dyer (ed.). The experimental biology of ferns. London, Academic Press.

Page, C.N. 1979b. Experimental aspects of fern ecology. Pp. 551-589. In: A.F. Dyer (ed.) The experimental biology of ferns. London, Academic Press.

Pichi-Sermolli, R.E.G. 1996. Authors of scientific names in Pteridophyta. Kew, Royal Botanical Gardens.
Prado, J. 1998. Pteridófitas do Estado de São Paulo. Pp. 49-61. In: C.E.M. Bicudo \& G.J. Shepherd. (eds.). Biodiversidade do Estado de São Paulo: Síntese do conhecimento ao final do século XX - Fungos macroscópicos e plantas. São Paulo, FAPESP.

Prado, J. 2004. Pteridófitas do Maciço da Juréia. Pp 139-151. In: O.A.V. Marques \& W. Duleba (eds.). Estação Ecológica JuréiaItatins. Ambiente Físico, Flora e Fauna. Ribeirão Preto, Holos Editora.

Pryer, K.M.; Schuettpelz, E.; Wolf, P.G.; Schneider, H.; Smith, A.R. \& Cranfill, R. 2004. Phylogeny and evolution of ferns (Monilophytes) with a focus on the early leptosporangiate divergences. American Journal of Botany 91: 1582-1598.

Roos, M. 1996. Mapping the world's pteridophyte diversity systematics and floras. Pp. 29-42. In: J.M. Camus; M. Gibby \& R.J. Johns (eds.). Pteridology in Perspective. Kew, Royal Botanic Gardens.

Salino, A. 1996. Levantamento das pteridófitas da Serra do Cuscuzeiro, Analândia, SP, Brasil. Revista Brasileira de Botânica 19: 173-178.

Salino, A. 2002. New species and combinations in Thelypteris subg. Goniopteris (Thelypteridaceae). Brittonia 54: 331-339.

Salino, A. \& Joly, C.A. 2001. Pteridophytes of three remnants of gallery forests in the Jacaré-Pepira river basin, São Paulo State, Brazil. Boletim do Herbário Ezechias Paulo Heringer 8: $5-15$.

Salino, A.; Silva, S.M.; Dittrich, V.A.O. \& Britez, R.M. 2005. Flora Pteridofítica. Pp. 85-101. In: M.C.M. Marques \& R.C. Britez (orgs.). História Natural e Conservação da Ilha do Mel. Curitiba, Editora UFPR.

Sehnem, A. 1979. Aspidiáceas. Pp. 1-356. In: R. Reitz (ed.). Flora Ilustrada Catarinense. Itajaí, Herbário Barbosa Rodrigues.

Simabukuro, E.A. 1994. Lista de pteridófitas da mata ciliar da Reserva Biológica de Mogi Guaçu, SP. Insula 23: 91-98.

Smith, A.R.; Pryer, K.M.; Schuettpelz, E.; Korall, P.; Schneider, H. \& Wolf, P.G. 2006. A classification for extant ferns. Taxon 55: 705-731.

Smith, A.R.; Kreier, H.P.; Haufler, C.H.; Ranker, T.A. \& Schneider, H. 2006. Serpocaulon (Polypodiaceae), a new genus segregated from Polypodium. Taxon 55: 919-930.

Sylvestre, L.S. 1997. Pteridófitas da Reserva Ecológica de Macaé de Cima. Pp. 41-52. In: H.C. Lima \& R.R. Guedes-Bruni (eds.). Serra de Macaé de Cima: Diversidade Florística e Conservação em Mata Atlântica. Rio de Janeiro, Instituto de Pesquisas Jardim Botânico do Rio de Janeiro.

Tryon, R.M. \& Tryon, A.F. 1982. Ferns and allied plants with special reference to Tropical America. New York, SpringerVerlag.

Versão eletrônica do artigo em www.scielo.br/abb e http://www.botanica.org.br/acta/ojs 